\title{
Research on the Shape of Automobile Face Based on Cultural Translation
}

\author{
Xueyuan Zhang ${ }^{1 a}$, Liqing Huang ${ }^{2 b^{*}}$, Mingzhu Li $^{3 c}$ \\ ${ }^{1}$ School of Art, Jiangsu University, Zhenjiang, Jiangsu \\ ${ }^{2}$ School of Art, Jiangsu University, Zhenjiang, Jiangsu \\ ${ }^{3}$ School of Art, Jiangsu University, Zhenjiang, Jiangsu
}

\begin{abstract}
Based on the concept of cultural translation, aiming at the application status and problems of traditional cultural elements in automobile styling design, analyzing the mapping relationship between traditional cultural levels and cultural creative design elements, and constructing a cultural translation model. Sort out the process and method of extracting typical cultural elements from the material layer, organization layer and concept layer of traditional culture and transform them into detail points, characteristic lines and modeling surfaces, and analyze and summarize the existing automobile face design cases.
\end{abstract}

\section{Introduce}

Automobile design is a science that combines cultural language and technical language. The automobile as a symbol is also a manifestation of deep cultural materialization ${ }^{[1]}$. Throughout the developed countries in the automotive field in the world, their automotive design has formed a unique design style. The precision and meticulousness of German cars, the romantic elegance of French cars, the ruggedness of American cars, the exquisiteness of Japanese cars, the enthusiasm and agility of Italy cars, and the simplicity and lightness of Nordic cars are undoubtedly influenced by its unique national cultural spiritual connotation. China's automobile industry is in a critical period. In addition to a solid industrial foundation, it is also necessary to give automobiles with cultural characteristics and cultural connotations. Chinese culture has a long history, and its cultural translation and design integration in automobile styling design can promote the shaping of national automobile brands and the nationalization of Chinese culture.

\section{Cultural Translation}

"Transliteration" is a vocabulary in the category of linguistics, which refers to the special translation act of translating a character into another character through a medium language ${ }^{[2]}$ "Cultural translation" is a process of extracting, designing, expressing, reorganizing and disseminating traditional culture based on the concept of translation. Design, interpret and translate traditional culture, explore more possibilities, and finally realize the output of cultural value ${ }^{[3]}$, as shown in Figure 1.

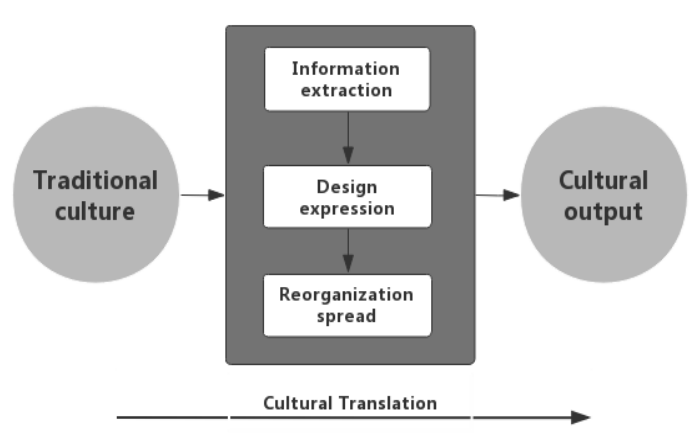

Figure 1: Graphical Analysis of Cultural Translation

\section{Translation of Traditional Culture in Automobile Modeling Design}

As the essence of Chinese culture, Chinese elements continue to this day. They are the inheritance and refinement of the Chinese nation's culture. They symbolize the unique characteristics of the Chinese nation, with both concrete physical manifestations and intangible ideologies ${ }^{[4]}$. The car styling design that integrates traditional Chinese cultural elements is still a cultural and creative product in essence. Culture and creativity are abstract concepts. The car is a concrete carrier, and the three are interdependent ${ }^{[5]}$. Therefore, the integration of Chinese traditional cultural elements into the car styling design aims to make car design more story-telling and cultural connotation, which is an extension of culture and has the nature of promoting cultural transmission.

\footnotetext{
a1826354090@qq.com

b*31475083@qq.com

c50884420@qq.com
} 


\subsection{The Way of Integration of Traditional Cultural Elements in the Design of Automobile Face}

The car's face design focuses on the lights, grilles, hoods, fog lights, bumpers, and outer steering. In car face design, the fusion expression of traditional cultural elements can be divided into two categories: cultural application and cultural translation according to the way of fusion, as shown in Figure 2. The application of culture is based on the research of automobile styling characteristics, using methods such as painting or engraving to directly apply culture to the surface of the product, without truly digging into the connotation and applicability of culture, and it will inevitably cause cultural elements in this process As a result of the misuse or improper use, users cannot truly understand the application meaning of culture in the process of perception; cultural translation is the application of traditional cultural elements determined from the design needs. It takes cultural elements as the starting point to target cultural elements. Deconstruction is carried out to obtain the cultural modeling semantics that conforms to the characteristics of the automobile front face shape, and then the automobile design rules are combined to shape each part, so as to obtain the automobile front face shape full of cultural meaning and connotation. Whether it is cultural application or translation, it is a rethinking of the modern application of traditional cultural elements, but cultural translation can obviously be more deeply integrated into the car styling design, awakening users' cultural identity.

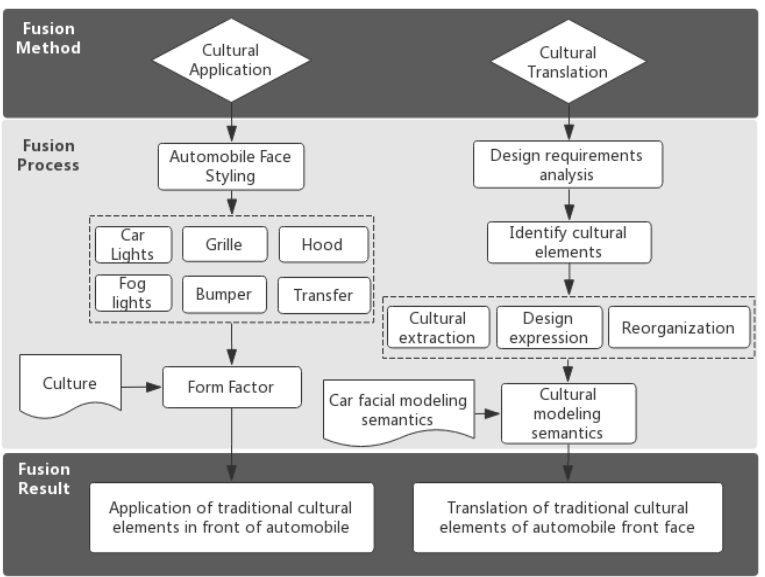

Figure 2: The car front face shape design and traditional culture integration method

\subsection{Cultural fusion in automobile styling design}

Only on the basis of traditional ideology and culture, Chinese car design combined with Chinese lifestyle and way of thinking can meet the functional needs of Chinese users at the root and have the style and characteristics of Chinese cars $^{[6]}$. In recent years, domestic and foreign automobile companies are trying to explore the application of Chinese cultural elements, such as Ferrari 599GTB China Limited Edition Song Dynasty Ge Kiln Porcelain Edition, Jeep Wrangler Dragon Collector Edition, Ferrari 458 Chinese Dragon, Rolls Royce China
Red, Bentley Mulsanne The Four Seasons Collection, Bugatti blue and white porcelain supercars, Mazda Chinese philosophy, BMW 7 series customized version, etc. Domestic car companies such as Hongqi use palace lantern elements and structural styling elements of Tiananmen Square, QiRui ARRIZO ancient Chinese bridge roof, Brilliance's Dan Fengyan car lamp shape, and BYD Dynasty series' long face shape, Geely water ripples and wishful shapes, etc.

Table 1: The fusion of Chinese traditional cultural elements in car design

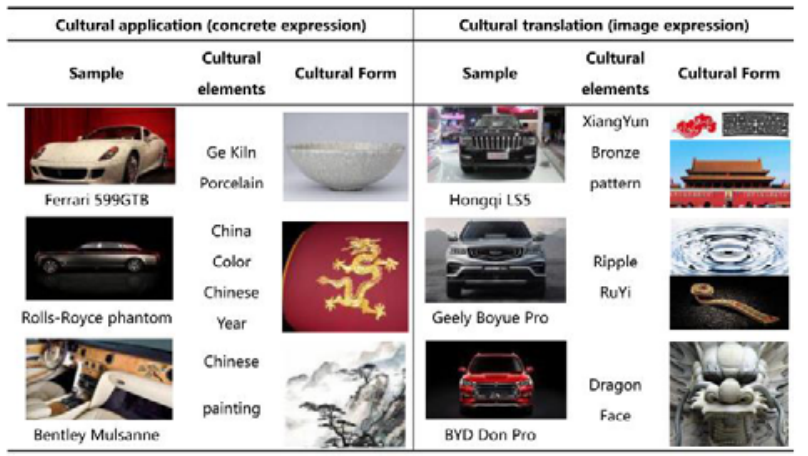

As shown in Table 1, the above application cases of Chinese traditional cultural elements are classified according to the way of cultural integration. Cultural application is the concrete expression of culture, a simple application of traditional Chinese cultural elements, and the cognitive flow of traditional Chinese cultural elements On the surface, this was also a common method used by foreign car companies in the early years to develop the Chinese market to increase sales; with the improvement of national cultural confidence, Chinese car companies pay more attention to the expression of their inner meaning when exploring the application of Chinese elements, which is also in line with The artistic conception of "Articles Carrying Tao" in traditional Chinese creations ${ }^{[7]}$ is the cultural translation of the application of cultural image expression.

\section{The level of cultural translation in car face design}

The cultural elements in car styling design are mainly the expression and extension of cultural connotations. The car styling of Chinese charm should have cultural concepts and language characteristics, so the analysis and layering of cultural elements is particularly important. In The Scientific Theory of Culture translated by X. T. Fei ${ }^{[8]}$ and others, culture is divided into three levels with a pyramid model, namely the artifact level, the organization level, and the concept level; Leong and Clark $^{[9]}$ proposed to study culture The framework and integrated design theory divide the cultural space into three levels: external, intermediate, and internal. The external level mainly includes form, material, the intermediate level includes behavior habits, life customs, and the internal level includes meaning, consciousness, spirit; $\operatorname{Lin}^{[10]}$ refer to the factors that need to be considered in product design as cultural and creative 
products design attributes include: External (Shape) layer, Middle (Associative) layer, and Inner (Spiritual) layer. On this basis, the three cultural levels were separately integrated into the design attributes of cultural products. He described the middle level of the cultural hierarchy as an invisible design feature that combined with life customs, which is a design element that cannot be seen through vision ${ }^{[11]}$.

Based on the understanding of cultural levels and cultural design attribute levels, taking into account the particularity of car face design, design attributes such as operability, practicality, and functionality are relatively weakened in the middle behavior level, so the middle layer of car face shape The extension of translation is extended to the intermediate correlation layer of morphological structure. The middle correlation layer mainly refers to the composition relationship between the points, lines, and faces in the face of the car, which conforms to the composition principle and aesthetic feelings of traditional Chinese culture. Construct a graphical analysis of the cultural level of the car front face design as shown in Figure 3.

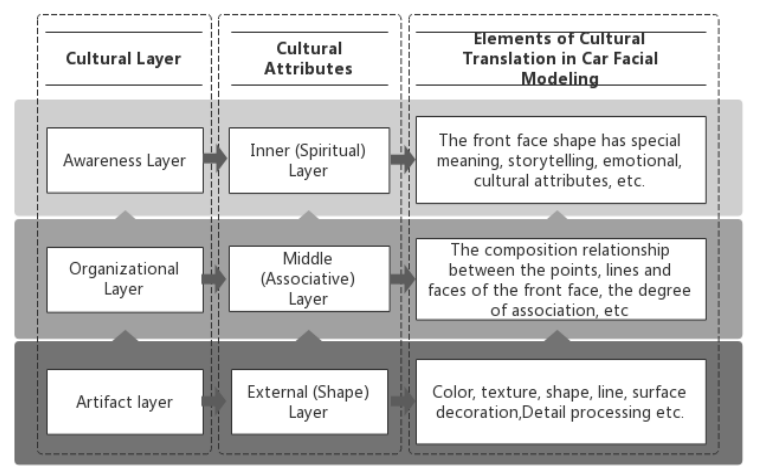

Figure 3: Graphical analysis of cultural levels in automobile face design

\subsection{Artifacts layer translation of traditional cultural}

The artifact layer in the cultural level is the human application and understanding of material, which is usually reflected in the fields of clothing, food, housing, and transportation related to people [12]. The cultural elements in the artifact layer include some obvious visual elements such as material texture, color texture, decorative symbols, artifact modeling, and detail processing.

When translating the cultural artifacts in the design of the front face of a car, first determine the design positioning and design goals. For example, the styling elements of commercial vehicles are biased towards atmosphere and safety, and the styling elements of offroad vehicles are biased towards strength and stability. Secondly, determine the prototype of the typical cultural elements, design a questionnaire to screen and evaluate the cultural elements that meet the image conditions in the typical cultural element resource library, and screen the cultural elements that meet the design requirements. Then extract and process the translation information of the selected traditional cultural elements. The designer needs to extract and process the shape, characteristic lines, abstract or concrete elements of the selected elements. Based on the characteristics of the front face of the car, it will have The elements of similar features are disassembled ${ }^{[13]}$, and the abstract modeling features are integrated into the front face of the car according to the basic principles of graphic design ${ }^{[14]}$. The material layer translation of traditional culture in the car front face design is shown in Figure 4.

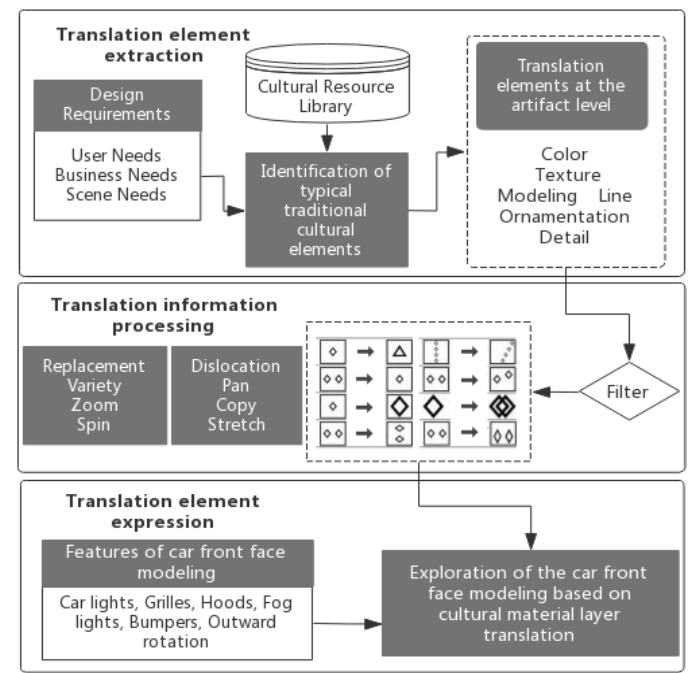

Figure 4: The artifact layer layer translation of traditional culture in automobile facial modeling

In our country, the image shaping of many selfdesigned car brands is a typical case of translating the material layers of traditional culture into product modeling language. For example, Geely Automobile has combined Chinese traditional cultural elements in the process of continuous exploration of its brand image to form a unique and highly recognizable facial shape. In the Geely family of products, the most recognizable grille part of the face uses the ripple shape of the water element and the shape of the Ruyi, and combines it with the car face shape rules to abstract and condense it. When consumers understand this design element, they can associate traditional cultural visual features with product semantics, and create associations. Geely automobile brand modelling translation is shown in Figure 5.

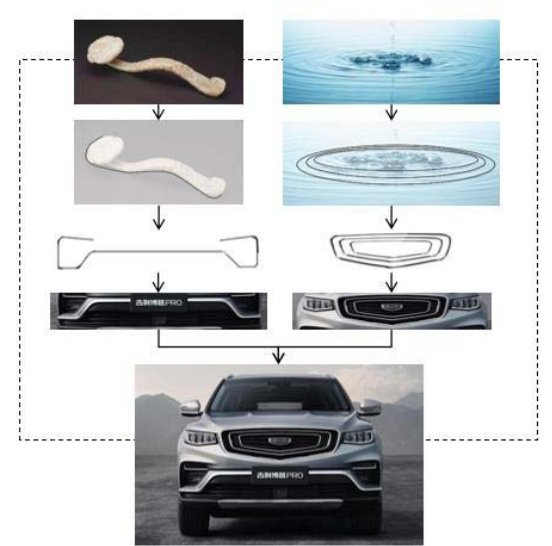

Figure 5: The artifact layer translation of Geely car's face shape 


\subsection{Organizational layer translation of traditional culture}

The organizational layer in the cultural hierarchy, that is, the middle layer of culture, is a concentrated expression of people's customs, lifestyles, and emotional expressions, and affects people's lifestyles and habits ${ }^{[15]}$. At the same time, the organizational layer is also the fusion part of the conceptual layer and the material layer, forming systems, rules, and laws ${ }^{[16]}$. Since the key to car face modeling is the three-dimensional modeling of the relationship between points, lines, and faces, it is highly regular and recognizable. Therefore, the organizational layer translation of car front face modeling design should be studied on the basis of typical cultural elements. The structural relationship and proportions of the styling form and the organizational structure of the details are to interpret the aesthetic essence of Chinese traditional cultural styling, and pay attention to its relevance and applicability to the car's detailed characteristic lines. For example, the styling ratio, hierarchical relationship, symmetry principle and depth rule in architectural culture can provide certain cultural styling inspiration for car facial sculpting.

Translating at the cultural organization level in the car styling design, in addition to following the formal beauty rules of typical cultural elements, the cognitive image of the target user must also be considered. First, consult relevant literature, collect image-perceived vocabulary and screen and classify it, invite relevant design experts and scholars to screen, construct anti-meaning semantic vocabulary pairs, use semantic difference method for quantitative analysis, and obtain typical examples from target user groups through scale method The image perception evaluation of cultural elements determines the description vocabulary of style characteristics ${ }^{[17]}$. Based on the determined image vocabulary, combined with the object layer to translate abstract and simplified modeling forms, the integrity and cultural recognition of automobile modeling design are improved. The organizational layer translation of traditional culture in automobile styling design is shown in Figure 6.

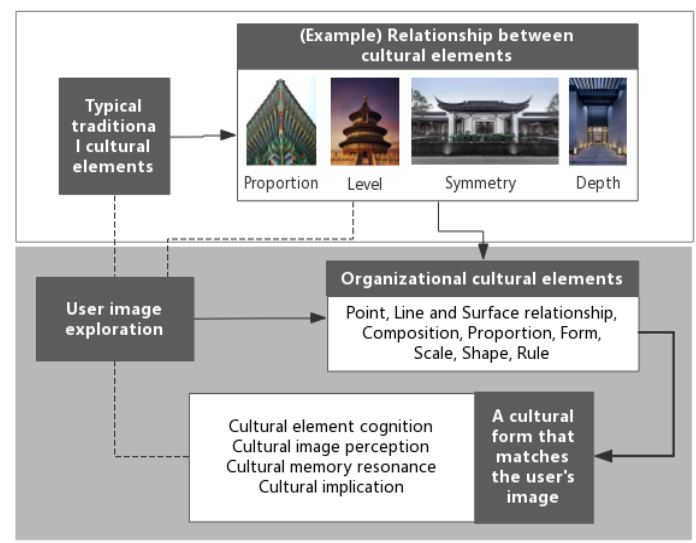

Figure 6: Organizational Translation of Traditional Culture in Car Facial Design

As shown in Figure 7, the facial styling of the Geely Boyue Pro and BYD Song Pro models has the material layer translation of traditional culture in the modeling. Among them, Geely Boyue uses water element ripples and Ruyi shapes in the design process. However, the downward-trending water drop ripple shape semantics is visually cut off by the horizontal lower grille shape at the bottom, presenting a sense of visual disharmony; BYD Song's face shape uses the "Dragon Face" shape semantics, the visual impetus of the lines created by the face is integrated as a whole, giving a domineering feeling. At the same time, the "Dragon Beard" chromeplated line extending from the front grille adds a sense of majesty and domineering compared with the huge "Dragon mouth". Therefore, the organizational layer translation of cultural elements in automobile styling design needs to be based on the translation of the material layer, follow the formal beauty rules of typical cultural elements, and pay attention to the relevance and integrity between details, characteristic lines, and modeling surfaces.

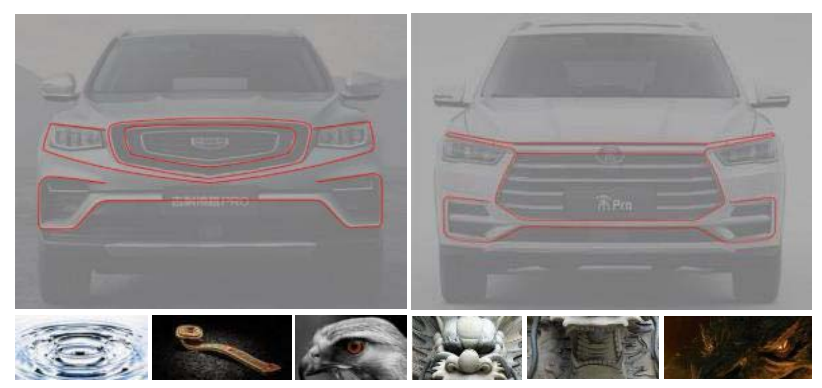

Figure 7: Geely (Boyue Pro)-BYD (Song Pro) face shape organization layer translation comparison

\subsection{Awareness layer translation of traditional cultural}

The conceptual layer in the cultural level, that is, the deep expression of culture, is the philosophical thought, aesthetic preference, and humanistic concept that have been inherited from traditional culture. Conceptual translation can help visual aesthetics to be raised to the level of cultural connotation. Since the cultural connotation of the conceptual level does not have a concrete material form and organizational form, but exists in an abstract, introverted, and implicit form, the translation of the conceptual level needs to be based on the material culture and the organizational level of the material culture and the organizational level. Above, fusion of Chinese traditional creation aesthetics for expression. The conceptual level of Chinese traditional culture includes philosophical thinking, moral ethics, humanistic spirit and aesthetic principles. Users need to use past experience, experience and habits in the cognitive process, or through cultural propaganda, story construction and scene construction. To arouse the resonance of the user's spiritual level, to gain cultural recognition, to form the inertia of cultural thinking, and to enhance the cultural self-confidence in the car styling design. The conceptual translation of traditional culture in automobile styling design is shown in Figure 8. 


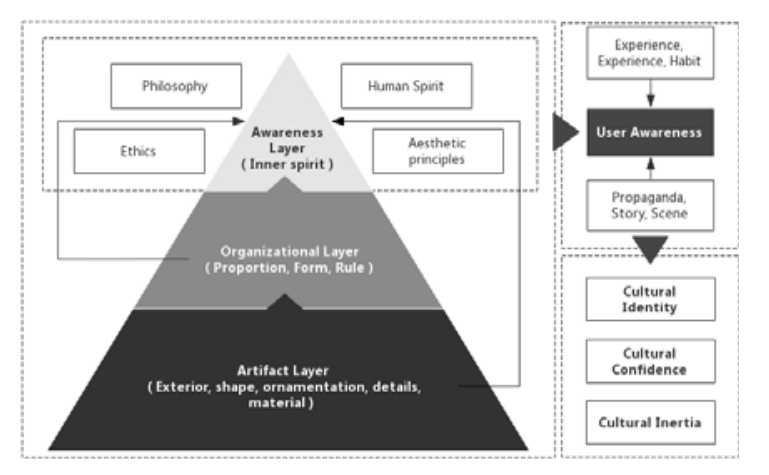

Figure 8: Conceptual translation of traditional culture in the design of automobile front face

As shown in Figure 9, Hongqi H9 is taken as an example to analyze the translation method of the inner spirit of the conceptual layer of Chinese traditional culture. Hongqi H9 combines user needs and contemporary aesthetic trends to innovate and recreate Chinese elements. The design of the front face of Hongqi H9 refined the imagery of "Alpine waterfalls, Mainstay" and "Dreams Agitating, Flying with Wings" in the translation of artifacts, and designed the new red flag car logo, straight waterfall grille and U-shaped penetration Car lights; in the process of organizational translation, based on the design philosophy of "Noble, Extreme, Artistic Conception" and family design genes, fully deconstruct the aesthetic sense of form and use the traditional Chinese aesthetics of silver. The grille design and the penetrating driving lights are harmoniously integrated into the front face shape. On the basis of the two, visual expression and artistic conception are used to make the front face of the car show the "modest gentleman" who is both internal and external, making people look at it at a glance. It will produce an indescribable sense of luxury and solemnity.

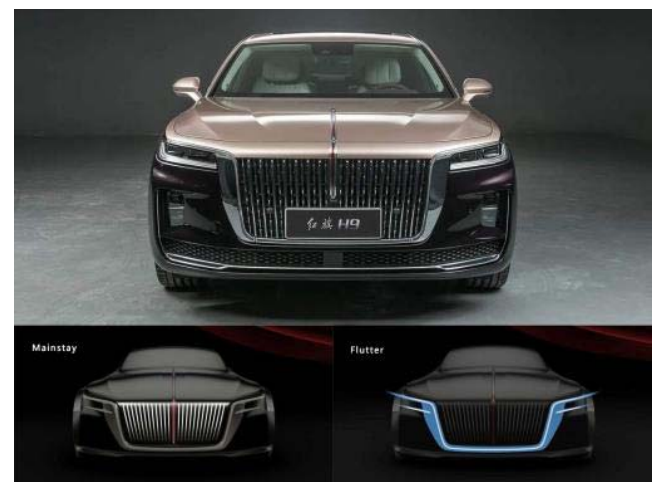

Figure 9: Hongqi H9 facial styling cultural concept layer translation

To sum up, the translation method of traditional Chinese culture in car face design requires full consideration of three levels in the cultural hierarchy: the extraction of typical cultural elements at the artifact level, the construction of the structural relationship of cultural elements at the organizational level, and the deep-level culture at the conceptual level. Connotation resonance. In the process of constructing the cultural translation model, we must not only seek for the typical elements that are suitable for the translation of automobile styling design, conform to the Chinese aesthetics and broadly represent Chinese culture, but also pay attention to the spiritual connotation contained in the culture itself, and carry out the Chinese culture from a deep level.

\section{Conclusion}

With the continuous development of China's automobile industry, the cultural and national characteristics of Chinese automobile design will become more and more obvious. Consumers will have higher and higher cultural connotations and artistic aesthetic requirements for automobile design, and there will be more in the future. The car styling design form of Chinese traditional cultural elements and spiritual connotation. The purely concrete expression of cultural elements can no longer meet the aesthetic needs of the market. It can combine the material, organization, and spiritual elements of traditional culture to translate traditional culture from the shallow to the deep, from the surface to the inside to the car design, and promote the national car brand building and widespread dissemination of Chinese culture.

\section{ACKNOWLEDGMENTS}

1.This work is supported by the University-Industry Collaborative Education Program, China (Grant No.201902177003)

2.This work is supported by the 19th batch of student research projects of Jiangsu University (Grant No.19C594)

\section{REFERENCES}

1. Huang Zhiqing. Modern automobile design and traditional culture $[\mathrm{J}]$. Art and Design (Theory), 2010(01):186-188.

2. Zhang Hao. The Application of Cultural Translation in the Context of Chinese Contemporary Art[J]. Art Education, 2013(10):28-29.

3. Liu Xu. Traditional cultural APP design based on the concept of cultural translation[J]. Packaging Engineering, 2020.

4. Ma Yuan, Zhang Jie. Application of Chinese Elements in Packaging Design[J]. Packaging Engineering, 2007, 28(10):218-220.

5. Wang Chaoxia, Yan Zexue. Research on Cultural and Creative Product Design Based on User Empathy[J]. Packaging Engineering, 2019(16):249253.

6. Zhang Xun. Research on the Application of Chinese Traditional Elements in Automobile Modeling Design Based on Emotional Theory[D].

7. Yang Xianyi, Zhou Yunfei, Wang Qin. On the Thought of "Usage to Carry Tao" in Traditional Chinese Creation[J]. Theory Monthly, 2006(12):5558. 
8. (English) Malinowski; Translated by Fei Xiaotong. Cultural Theory[M]. China Folk Literature and Art Publishing House, 1987.02.

9. Leong B D, Clark H. Culture-Based Knowledge Towards New Design Thinking and Practice-A Dialogue[J]. Design Issues, 2003, 19(3):48-58.

10. WU T Y, CHENG H, LIN R. The Study of Culture Interface in Taiwan Aboriginal Twin-Cup[J]. In Hci International, 2005(4): 22-27.

11. Lin R, Cheng R, Sun M X. Digital Archive Database for Cultural Product Design[M]// Usability and Internationalization. HCI and Culture. Springer Berlin Heidelberg, 2007.

12. Ma Jun. Exploring the Value of Chinese Traditional Cultural Semiotics[J]. Literary Circles (Theory Edition), 2011(11):227-228.

13. Wang Weiwei, Hu Yukun, Jin Xin, et al. Research and application of traditional cultural design element extraction model[J]. Packaging Engineering, 2014, 035(006): 73-76.

14. Zhao Danhua, He Renke, Tan Hao, et al. Semantic acquisition and expression of automobile brand style[J]. Packaging Engineering, 2013, 034(010):2730,61 .

15. Hao Jin. On the Use of Traditional Cultural Elements in Interactive Media Design[J]. The House of Drama, 2015(24):266-266.

16. Xue Wangxiang. Research on the characteristics of Guangzhou's urban features based on the theory of cultural levels[D].

17. Zhang Linghao. The semantic meaning of products[M]. China Construction Industry Press, 2009. 